\title{
Lo que todo profesor necesita saber sobre el razonamiento clínico*
}

\author{
What every teacher needs to know about clinical reasoning
}

Kevin W. Eva. Universidad de McMaster, Hamilton, Notario, Canadá

Contexto: Una de las tareas nucleares que se asignan a los profesores clínicos es capacitar a los estudiantes para escoger entre un grupo de características que presenta un paciente y asignar con precisión una etiqueta diagnóstica con el objetivo final de desarrollar una estrategia de tratamiento apropiada. Durante los últimos 30 años se ha debatido considerablemente en el seno de la literatura en Educación en Ciencias de la Salud, sobre el modelo que mejor describe cómo los clínicos expertos generan decisiones diagnósticas. Propósito: El propósito de este ensayo es proporcionar una revision bibliográfica de la investigación sobre razonamiento clínico. Los puntos fuertes y débiles de las diferentes aproximaciones del razonamiento clínico se examinarán usando una de las principales divergencias se da entre los modelos de estrategia de razonamiento analítico (p.ej., consciente / controlado) versus estrategias de razonamiento no analítico (p.ej.,

\footnotetext{
* Este artículo es una traducción de Maria Nolla Domenjó del artículo original publicado en Medical Education: Eva, W.K. What every teacher needs to know about clinical reasoning. Medical Education 2004;39:98-116. Educación Médica agradece la amabilidad de Blackwell Publishing Ltd por facilitar los derechos de traducción de este artículo.
}

\section{Correspondencia:}

Kevin W. Eva

Program for Educational Research and Development

Department of Clinical Epidemiology and Biostatistics

T-13, Room 101

McMaster University

Hamilton, Ontario, Canada

L8S $4 \mathrm{~K} 1$

Tel.: (905) 525-9140 ext. 27241

Fax: (905) 572-7099

E-mail: evakw@mcmaster.ca inconsciente / automático). Como marco de referencia orientativo.

Discusión: Trabajos recientes sugieren que los profesores clínicos deberían destacar la importancia de ambas formas de razonamiento, dando como resultado la capacitación de los estudiantes para ordenar los procesos de razonamiento de manera flexible y específica de contexto. Se extraen las implicaciones específicas para los profesores clínicos de primera línea.

Palabras clave: educación médica, pregrado, métodos; competencia clínica, educación clínica; toma de decisiones; métodos de enseñanza; revisión.

Context: One of the core tasks assigned to clinical teachers is to enable students to sort through a cluster of features presented by a patient and accurately assign a diagnostic label, with the development of an appropriate treatment strategy being the end goal. Over the last $\mathbf{3 0}$ years there has been considerable debate within the Health Sciences Education literature regarding the model that best describes how expert clinicians generate diagnostic decisions.

Purpose: The purpose of this essay is to provide a review of the research literature on clinical reasoning for frontline clinical teachers. The strengths and weaknesses of different approaches to clinical reasoning will be examined using one of the core divides between various models (that of analytic (i.e., conscious/controlled) versus non-analytic (i.e., unconscious/automatic) reasoning strategies) as an orienting framework.

Discussion: Recent work suggests that clinical teachers should stress the importance of both forms of reasoning, thereby enabling students to marshal 
reasoning processes in a flexible and context-specific manner. Specific implication are drawn from this overview for clinical teachers.

Key words: education, medical, undergraduate/methods; clinical competence/education; decision making; teaching/methods; review literature.

\section{INTRODUCCIÓN}

A las 2:00 de la madrugada, un hombre trajo a su esposa de 43 años a urgencias a causa de distrés respiratorio agudo. La disnea había aparecido de repente a las 11:00 PM despertando a la paciente. Sentía náuseas y vomitó una pequeña cantidad de bilis. Se quejaba de dolor torácico retroesternal que empeoraba con la respiración profunda. Durante unos días había expectorado pequeñas cantidades de sangre. Durante cuatro días se había sentido mal y tenía dolor de garganta y congestión de senos que se resolvió. En los últimos días se había quejado en diversas ocasiones de fiebre y escalofríos. La noche anterior se despertó con opresión en el pecho, pero remitió al cabo de poco tiempo. Había antecedentes de bronquitis ${ }^{1}$.

¿Cuál es el diagnóstico más probable? De la misma manera que en una historia de misterio determinar “¿quién lo hizo?”, el reto diagnóstico implica considerar cada pieza de información dis- ponible y determinar la explicación más plausible para el patrón presentado. Hacer esto no es una tarea sencilla. A menudo supone (a) observación cuidadosa, (b) adecuada obtención de la información histórica, (c) realización precisa de las maniobras físicas, (d) generación de hipótesis, (e) reconocimiento de la relación entre cada pieza de información y cada hipótesis, y (f) tratar de confirmar / descartar hipótesis a base de solicitar adecuadamente las pruebas diagnósticas. A diferencia de un lector de historias de misterio, el clínico a menudo se enfrenta a la tarea añadida de determinar si, cuando y cómo se recogerán varias piezas de información.

El reto a que se enfrentan los profesores clínicos es quizás aún mayor. Los profesores clínicos, no sólo deben ser capaces de realizar todas las tareas del párrafo anterior, sino que también deben encontrar una manera de comunicar su conocimiento y estrategias de razonamiento a los diagnosticadores novatos de manera que nutra la propia experteza del alumno. A lo largo de la última mitad de siglo se ha ido evidenciando que la habilidad para hacer esto está relacionada con, pero es distinta de, la experteza dentro del área de contenido a enseñar ${ }^{2}$. El mantenimiento de la experteza en la docencia clínica requiere, en parte, una comprensión de las estrategias que usan los clínicos expertos, a menudo inconscientemente, para razonar el diagnóstico en presentaciones de casos como el del inicio de este artículo $^{3}$. Al reto del profesor clínico hay que añadir el hecho de que los mecanismos psicológicos subya-

\section{Cuadro 1: Visión General}

\section{Qué se conoce sobre este tema}

Los modelos tradicionales de toma de decisiones clínicas proporciona descripciones insuficientes sobre la manera en que los clínicos razonan; los procesos no analíticos (por ejemplo, reconocimiento de patrones), juegan un papel relevante, aunque pueden introducir un sesgo en todos los niveles de esperteza.

\section{Qué aporta este artículo}

Este artículo orienta a los profesores clínicos hacia la necesidad de enseñar a los estudiantes estrategias de razonamiento múltiples. Intenta reconceptualizar, el constructo de experteza hacia una entidad amorfa que podría ser mejor definida como flexibilidad en relación a las maneras en que la soluciones a los problemas clínicos pueden ser alcanzadas.

\section{Sugerencias para posteriores investigaciones}

Se requieren estudios adicionales sobre la manera óptima en que varias estrategias de razonamiento puedan ser transmitidas a los estudiantes de medicina, sobre los factores de contexto que influencian la coordinación de los procesos analíticos y no analíticos, y sobre la naturaleza de la flexibilidad mostrada por los expertos. 
centes a este tipo de tendencias de razonamiento, no siempre son accesibles por introspección ${ }^{4}$.

Este artículo se ha escrito para proporcionar una revisión de la bibliografía sobre razonamiento clínico para los profesores clínicos. $\mathrm{Al}$ hacer esto, se proporcionarán pocos detalles relativos a los métodos de investigación que llevan a cada conclusión, pero se proporcionarán las referencias necesarias para los interesados. La atención se pondrá más bien en la comprensión actual de la manera en que los clínicos resuelven retos diagnósticos y las implicaciones que surgen de esta comprensión. La discusión se centrará en las técnicas de instrucción para maximizar la probabilidad de que los estudiantes tengan éxito al resolver problemas médicos y en las estrategias para valorar con precisión si los estudiantes han desarrollado de hecho las competencias requeridas. Para empezar realizaremos un examen cuidadoso de cómo se podría resolver el problema diagnóstico que abría este artículo.

\section{¿CUÁL ES EL DIAGNÓSTICO MÁS PROBABLE (Y CÓMO SE HA LLEGADO A ALCANZARLO)?}

¿Cuál es el diagnóstico más probable ( y cómo se ha llegado a alcanzarlo)?

No es necesario ir muy lejos para reconocer que los educadores médicos tradicionalmente se han centrado en lo que se conoce como modelos "analíticos" de razonamiento clínico; modelos que suponen que un análisis cuidadoso de la relación entre signos y síntomas y diagnósticos es el sello de la experteza clínica. Por ejemplo, el manual "Principios de
Medicina Interna" de Harrison presenta el distrés respiratorio como una indicación tanto de neumonía como de tromboembolismo pulmonar (TEP). En el caso anterior, las características de dolor de garganta, náuseas, y vómito sugieren neumonía ${ }^{5}$, mientras que la característica de esputo sanguinolento sugiere $\mathrm{TEP}^{6}$. La implicación, en ambos casos, es que los rasgos característicos son claramente evidentes y que el razonamiento diagnóstico implica la comprensión de la relación entre las características detectadas y los desórdenes subyacentes. Generar una lista diferencial de diagnósticos relevantes y aplicar un algoritmo diagnóstico adecuado permite ponderar cada diagnóstico en términos de su probabilidad relativa ${ }^{6}$.

Los modelos de razonamiento clínico que incorporan el teorema de Bayes o análisis de regresión son los que mejor representan esta forma de razonamiento $^{7,8}$. Brevemente, estos modelos asumen que los médicos son conscientes de la probabilidad a priori con que un diagnóstico en particular se pueda presentar y de la probabilidad condicional que asocia cada pieza de evidencia (p. ej., signos, síntomas, y pruebas diagnósticas) con los diagnósticos. El modelo matemático computa una valoración post-test de la probabilidad de cada diagnóstico considerado. Este proceso, ilustrado en la Figura 1, continua siendo promovido por individuos cercanos al movimiento de la Medicina Basada en la Evidencia ${ }^{9,10}$. Mientras que algunos han argumentado que el flujo de información hacia delante ilustrado en la Figura 1 (p.ej., razonamiento desde la evidencia hacia los diagnósticos) es el que mejor capta la esencia del razonamiento clínico "experto"11,12, las probabilidades subsiguientes detectadas también podrían usarse fácil-

Figura 1: Procesos analíticos en el razonamiento clínico

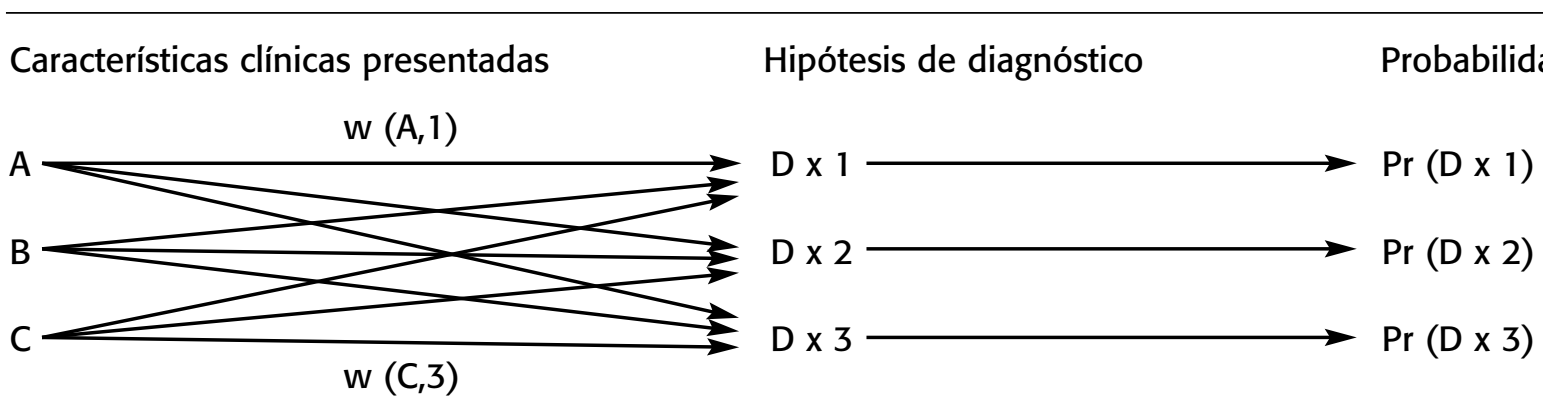

NOTA: Los diagnósticos únicos están indicados con números, las características clínicas con letras. Cada característica mantiene una relación única (p.ej., peso) con cada diagnóstico. La magnitud de los pesos se indica por el tamaño de la flecha. Dos (p.ej., peso de la relación entre la característica A y el diagnóstico 1) están etiquetadas. El resultado de combinar razones de base y pesos basados en la característica es señal de una probabilidad condicional $(\mathrm{Pr})$ para cada hipótesis diagnóstica que tiene en cuenta el grupo de características observadas. 
mente para dar feedback a la recogida y análisis de datos adicionales y el modelo permanecería como un modelo de procesamiento analítico siempre y cuando la noción de análisis cuidadoso se mantenga.

De hecho, los procesos analíticos se han hecho operativos de muchas maneras a través de diferentes modelos de experteza ${ }^{13,14,15}$. Sin embargo, en el corazón de cada enfoque está la creencia fundamental de que las reglas causales que unen características (p. ej., signos y síntomas) con categorías (p. ej., diagnósticos) pueden ser extraídos del mundo y que el desarrollo de la experteza en el razonamiento clínico consiste en el desarrollo y elaboración de reglas que sintonizan cada vez más con la realidad. Esta visión del razonamiento clínico sugiere que la tarea del educador es facilitar el desarrollo de dichas reglas. Como ejemplo, Elieson y Papa han mostrado un beneficio pedagógico al proporcionar a los estudiantes ayudas diagnósticas que describan explícitamente las relaciones probabilísticas entre características y síntomas ${ }^{16}$. Sin embargo, uno no necesita mantener una visión tan extrema de la utilidad de probabilidades explícitas para incorporar el coste de las estrategias de razonamiento analítico en su docencia clínica - teorías intuitivas del valor del razonamiento analítico se unen cada vez que un profesor clínico amonesta a un estudiante con "ser objetivo" y que considere cuidadosamente toda la evidencia disponible antes de generar hipótesis diagnósticas. No obstante, hay otro camino para resolver el problema descrito anteriormente, un camino que ha recibido atención creciente en los últimos 15 años. Como forma de ilustración, trate de diagnosticar el caso siguiente:
A las 2:00 de la madrugada, un hombre trajo a su esposa de 43 años a urgencias a causa de distrés respiratorio agudo. La disnea había aparecido de repente a las 11:00 PM despertando a la paciente. Sentía náuseas y vomitó una pequeña cantidad de bilis. Se quejaba de dolor torácico retroesternal que empeoraba con la respiración profunda. Durante unos días había expectorado pequeñas cantidades de sangre. Durante cuatro días se había sentido mal y tenía dolor de garganta y congestión de senos que se resolvió. En los últimos días se había quejado en diversas ocasiones de fiebre y escalofríos. La noche anterior se despertó con opresión en el pecho, pero remitió al cabo de poco tiempo. Había antecedentes de bronquitis.

Incluso los lectores clínicamente naifs reconocerán que un diagnóstico diferencial plausible para este caso incluye neumonía y tromboembolismo pulmonar. ¿Por qué? Porque este caso se ha encontrado antes (al principio de este artículo). Cuando se pregunta "cuánto es 120 dividido entre 10", la mayoría de nosotros puede responder rápidamente y sin esfuerzo "12". A menudo y de forma similar, los clínicos están en una posición en la que no necesitan "razonar" para nada. En algunos círculos se denomina reconocimiento de patrones, la forma más general del "razonamiento no analítico", ilustrado en la Figura 2, que esencialmente equivale a comparar el caso actual con los que se han encontrado en el pasado y utilizar estas experiencias pasadas para hacer juicios relacionados con la probabilidad de que cualquier caso pertenezca a una categoría diagnóstica

\section{Figura 2: Procesos no analíticos de razonamiento clínico}

\section{Características clínicas presentadas}

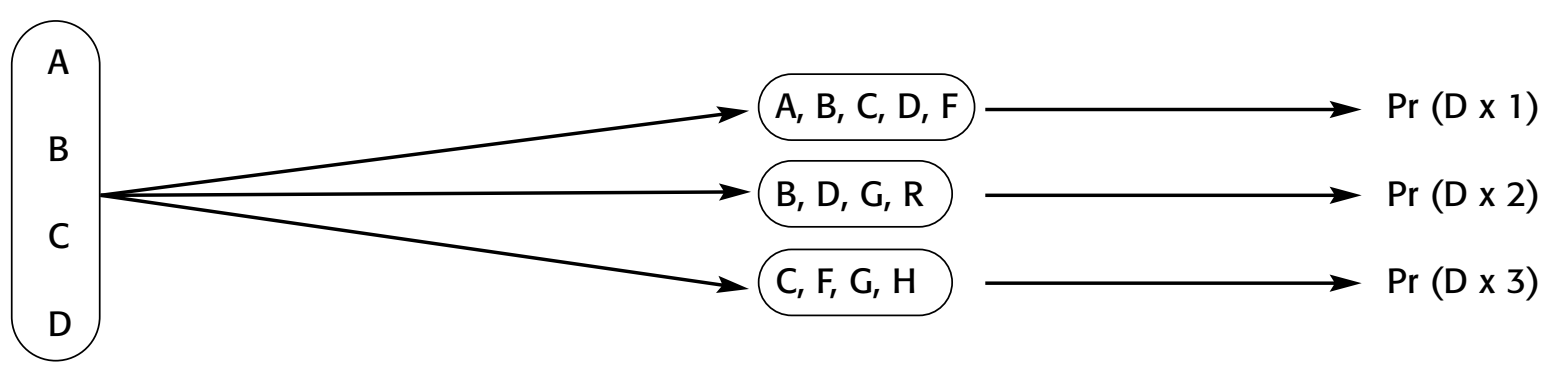

NOTA: Los diagnósticos únicos están indicados por números, las características clínicas por letras. Cada paciente (representado por rectángulos redondeados) se presenta con un grupo de características. Este grupo se compara (inconscientemente) con ejemplos encontrados en el pasado dando como resultado una probabilidad de ser asignado para cada hipótesis. La fortaleza de la correspondencia entre el paciente actual y las experiencias pasadas es variable tal como se indica por le tamaño de las flechas. 
particular $^{17,18}$. El ejemplo que se ha proporcionado aquí es un caso extremo donde todas las características se presentaron exactamente de la misma manera en ambas ocasiones, pero tal como se ilustra en la Figura 2, no es necesario que todas las características se correspondan en el mismo orden para que se identifique una correspondencia potencial.

Se ha propuesto la hipótesis que esta forma de "razonamiento" se da con suficiente automatismo y que frecuentemente sucede sin plena consciencia. A pesar de la tendencia que tenemos como humanos a dar explicaciones de nuestras acciones, en realidad, la fuente de nuestro comportamiento/decisiones nos es a menudo desconocida ${ }^{4}$. A pesar de que este hecho hace imposible asumir como válidas las respuestas obtenidas simplemente preguntando a los clínicos si usan y cuándo usan el reconocimiento de patrones, la evidencia de que los clínicos usan procesos no analíticos para llegar a las decisiones diagnósticas es indiscutible ${ }^{19}$. Por ejemplo, Brooks et al. han mostrado en una serie de estudios, en los que la precisión diagnóstica es mayor en los casos dermatológicos que son similares a casos que se han visto previamente que en relación a los casos cuyo aspecto es bastante distintos ${ }^{20}$. Además, Hatala et al. han informado de que incluso las características irrelevantes para el diagnóstico de un caso (p. ej., ser banquero) tienen un impacto sobre el diagnóstico de casos que se presentan subsecuentemente en los que la pieza de información irrelevante es similar ${ }^{21}$.

Se ha argumentado que la habilidad para usar bases no analíticas de toma de decisiones clínicas aumenta con la experteza y, como resultado, no se debería defender el uso del reconocimiento de patrones entre los estudiantes de medicina por miedo a las "potenciales consecuencias adversas". ${ }^{22, p .699 . ~ E n ~}$ un extremo, está el caso de los novatos totales que no tienen experiencia pasada de la que fiarse y, por lo tanto, son incapaces de utilizar estrategias de razonamiento basadas en la semejanza. No obstante, la realidad ha mostrado que la estrategia empleada incluso por los estudiantes de medicina más jóvenes es cualitativamente indistinguible de la de médicos expertos, ya que ambos grupos generan muy rápidamente hipótesis, presumiblemente basadas en parte en referencias no analíticas de experiencias anteriores ${ }^{23}$. Sin embargo, es más probable que los clínicos con más experiencia generen respuestas correctas, tal como se esperaría dado que tienen una base de datos mayor a la que referirse. Más directamente, siempre que se ha comparado explícitamente la ventaja de enseñar a los estu- diantes a razonar de manera analítica con la influencia de enseñar a los estudiantes a confiar en sus juicios no analíticos, la precisión diagnóstica ha sido como mínimo tan buena si no mejor en el grupo entrenado a utilizar razonamiento no analítico, incluso entre novatos relativos ${ }^{22}$. Las bases no analíticas del juicio no son inferiores a las formas más analíticas de razonamiento y los profesores clínicos deberían informar a sus estudiantes que la semejanza con situaciones pasadas puede servir como guía útil. El potencial para "consecuencias adversas" es un argumento demagógico dado que la responsabilidad final de la atención clínica recae típicamente en el profesor clínico, no en los estudiantes novatos.

\section{LA UNIÓN DE LAS ESTRATEGIAS DE RAZONAMIENTO ANALÍTICO Y NO ANALÍTICO}

Dicho esto, parece realmente ser cierto que la excesiva confianza en los enfoques no analíticos para el razonamiento clínico puede ser fuente de errores diagnósticos. Las primeras impresiones, aunque útiles, a menudo son incorrectas, incluso entre los clínicos expertos ${ }^{24}$. Los factores de contexto, como recibir una sugerencia diagnóstica, se han mostrado que disminuyen tanto (a) la probabilidad de que las características consistentes con diagnósticos alternativos sean identificadas ${ }^{25}$, como (b) el peso relativo asignado a características consistentes con los diagnósticos alternativos que se identifiquen $^{26}$. Eva ha proporcionado evidencia de que estos sesgos derivan de la excesiva confianza en los procesamientos no analíticos; la simple orden de listar explícitamente la evidencia presente en un caso (p. ej., una orden que se puede anticipar para favorecer un proceso más analítico) se ha visto que es suficiente para eliminar este tipo de sesgo ${ }^{27}$. Sin embargo, un factor crítico fue que el procesamiento analítico fuera llevado a cabo en relación temporal próxima a la realización del juicio diagnóstico real. ¿Dónde deja esto al profesor clínico? En primer lugar, hay que reconocer que estas dos formas de procesamiento no son mutuamente excluyentes. Es altamente probable que ambas formas de procesamiento contribuyan a las decisiones finales que se alcanzan en todos los casos (para ambos, novatos y expertos). El impacto de la semejanza, en algunos casos, será incitar una consideración analítica del caso actual que es análogo a los análisis que se rea- 
Figura 3: Un modelo combinado de razonamiento clínico
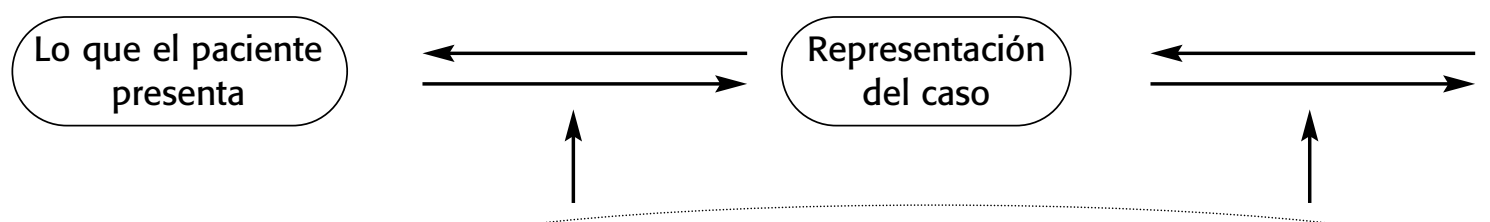

Hipótesis comprobada

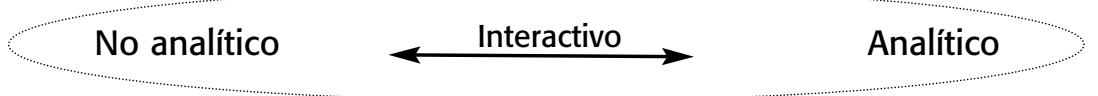

NOTA: Los modelos analíticos y no analíticos de las Figuras 1 y 2 se combinan en este modelo. Cada tipo de procesamiento se cree que interacciona con ambas representaciones mentales del caso que se presenta y las hipótesis que han surgido, pero en grados diferentes en función del contexto.

lizaron en un caso similar en el pasado. Como resultado, la forma óptima de razonamiento clínico debería ser considerada como un modelo adicional en el que ambos procesamientos, analítico y no analítico, juegan un papel. Un modelo de este tipo se ilustra en la Figura 3. En este modelo, el clínico forma una representación mental del caso a partir de la presentación de un paciente y esta representación mental lleva a probar la hipótesis lo cual, en la mayoría de los casos, conducirá la forma de hacer la historia clínica, el examen físico, y la solicitud de pruebas diagnósticas. Es importante ver que la dirección del razonamiento se ilustra procediendo en ambas direcciones; los resultados de comprobar la hipótesis influenciará la representación mental que mantiene el clínico y las representaciones mentales pueden influenciar la manera como se perciben los problemas del paciente. El flujo bidireccional se puede esperar tanto en novatos como en expertos ${ }^{28}$. Además, se debería subrayar que se espera que los procesamientos no analíticos dominen durante las fases iniciales al considerar un nuevo caso, mientras que el procesamiento analítico se espera que juegue un papel dominante en la comprobación de la hipótesis. Estas dos formas de razonamiento se deberían ver como muy interactivas, sin encontrarse a lo largo de un continuum, pero en cambio como contribuyentes complementarios a la precisión global del proceso de razonamiento clínico, cada uno influenciando al otro $29,30,31$.

Trabajos recientes proporcionan soporte práctico para este modelo ${ }^{32}$. Durante la enseñanza de novatos absolutos (estudiantes de Psicología) sobre el diagnóstico de electrocardiogramas (ECGs), a un grupo de participantes se les dio la instrucción de confiar en los sentimientos de semejanza (una base no ana- lítica del razonamiento clínico) durante las fases de práctica y examen, pero a evitar precipitarse a base de considerar explícitamente las características específicas presentes en el ECG (una estrategia analítica). Este grupo mostró una precisión diagnóstica más alta que los otros dos grupos, aquellos participantes que recibieron bien la instrucción no analítica, bien únicamente la instrucción analítica. La realización del diagnóstico de los dos últimos grupos era equivalente. Este resultado replica y amplia trabajos anteriores en los que instrucciones combinadas daban como resultado mayor precisión diagnóstica que las instrucciones puramente analíticas ${ }^{33}$. En ambos estudios parece claro que los estudiantes a los que se les proporcionó la instrucción de ser puramente analíticos se encontraron inundados en un torrente virtual de características clínicas, haciendo difícil reconciliar el patrón observado con una sola entidad diagnóstica. Además, otros trabajos sugieren que el fracaso en realizar una confirmación analítica tiene como resultado el cierre prematuro y el error diagnóstico, incluso entre los clínicos altamente expertos ${ }^{24}$. Para evitar ambos estados, los profesores clínicos deberían promover la combinación de ambas formas de razonamiento. Otras consideraciones de las implicaciones de esta visión se destacarán después de una breve nota sobre la estabilidad de estrategias diagnósticas específicas.

\section{SITUACIÓN ACTUAL DE LA RELACIÓN ENTRE LAS DOS ESTRATEGIAS DE RAZONAMIENTO}

De la descripción anterior del razonamiento clínico es fácil inferir la necesidad de los profesores clí- 
nicos de nutrir a los estudiantes para convertirse en "buenos solucionadores de problemas" o en "buenos coordinadores de los procesamientos analíticos y no analíticos". Estas visiones del proceso diagnóstico son obsoletas e imprecisas, a causa del sólido hallazgo de que la solución correcta de un problema clínico no predice de manera precisa la solución correcta de otro problema clínico, incluso dentro de una área de especialización ${ }^{34,35}$. La habilidad de razonamiento no es un "rasgo" que se pueda asignar a un individuo. Indudablemente algunos individuos diagnostican mejor que otros, pero los profesores clínicos deben reconocer que el contexto dentro del cual se trata un problema (p. ej., el "estado") tiene un impacto mayor sobre la precisión alcanzada y el balance óptimo entre la "estrategia" de razonamiento potenciales. "El contexto" incluye ambos factores de situación (p.ej., el contexto clínico y los casos que se han visto recientemente) y factores personales ( $p$. ej., la experiencia de los clínicos y los pensamientos actuales).

A pesar de los intentos de ofrecer a los estudiantes un curriculum uniforme, nunca dos estudiantes tienen exactamente las mismas experiencias. Estudiantes diferentes ven casos diferentes, reflexionan sobre aspectos diferentes de un caso dado, y sacan insights diferentes de este tipo de reflexiones. Cada una de estas diferencias impactará sobre la manera en que un estudiante individual enfoca un caso dado (p. ej., factores de situación específicos influenciarán la "estrategia de razonamiento" adoptada). Esta concepción basada en el estado de la práctica clínica quizá sea la razón más fundamental para asegurar que a los estudiantes se les proporciona múltiples estrategias que los podrían capacitar para trabajar con un problema clínico. En algunos casos es más probable que una dosis fuerte de reconocimiento de patrones produzca la solución correcta. En otros, se podría requerir una historia más completa, o la aplicación de un algoritmo diagnóstico, o la consideración de las ciencias básicas subyacentes a la patofisiología. Cuantas más herramientas tiene uno en su taller más probable es que una de las herramientas permita el cumplimiento de la tarea con éxito.

Con esto en mente, se hace improbable que cualquier constructo defina alguna vez plenamente el término "experto" en un dominio tan amplio como el razonamiento clínico. Como analogía, consideremos el desarrollo de la experteza en la lectura. Cuando realizaron un análisis del componente de habilidades de la capacidad de leer, Levy and Hinchley administraron series de once tests de lectura y hallaron que mientras los lectores pobres eran en promedio peores que los buenos lectores, cada lector individual tenía algunos puntos fuertes y algunos puntos débiles $^{36}$. El $56 \%$ de los buenos lectores tenían déficits en la realización de uno o más tests y el $58 \%$ de los lectores pobres mostraban habilidades superiores en uno o más tests. Por lo tanto, la experteza en el razonamiento clínico, probablemente debería ser considerada una entidad amorfa que capacita a los clínicos competentes para compensar las debilidades específicas de un caso concreto. Esto permite adaptarse a las demandas de la situación, utilizando de forma flexible (y a menudo inconscientemente) todo el armamento disponible.

\section{IMPLICACIONES PARA LOS PROFESORES CLÍNICOS}

En resumen, se ha debatido mucho en el seno de la literatura en educación médica con relación a la estructura de la experteza médica. Al final de los 70, Elstein, Shulman, y Sprafka presentaron el modelo hipotético-deductivo de razonamiento clínico, a saber, aquel en que delante de un caso nuevo, los médicos generan un conjunto de hipótesis que después usan para comprobar con los datos presenta$\operatorname{dos}^{37}$. Desde entonces se han desarrollado numerosos marcos de referencia de representación del conocimiento, pero la investigación realizada en los últimos 15 años ha cuestionado si algún marco de referencia probará ser correcto ${ }^{38}$. Aún más recientemente, se está empezando a acumular evidencia que sugiere un abordaje más comprehensivo para al enseñanza clínica, uno que incluye el reconocimiento de los beneficios de los dos enfoques del razonamiento clínico, analítico y no analítico, y pueda capacitar a los estudiantes para aprovechar lo mejor de ambos mundos ${ }^{32}$. Una conciencia adicional de la prevalencia de la especificidad de contexto ha subrayado la necesidad de proporcionar a los estudiantes un abanico de estrategias que los puedan posicionar mejor para adaptarse con flexibilidad a las demandas de la situación. Los párrafos que restan de este artículo darán una visión general sobre algunas de las implicaciones que surgen de esta comprensión actual en un esfuerzo para facilitar la reflexión sobre las técnicas pedagógicas actuales y estimular el desarrollo de nuevos abordajes.

En primer lugar y más importante, esta revisión subraya la importancia de enseñar entorno a ejem- 
plos. Cuanto antes empiecen los estudiantes a acumular una base de datos mental de casos, antes tendrán un fundamento firme sobre el que permitir la contribución de los procesos no analíticos. Esta idea no es nueva para los educadores médicos, es un principio fundamental de la buena pedagogía. No obstante, lo que es relativamente nuevo, es el reconocimiento de que pocos ejemplos complejos y elaborados, probablemente sean subóptimos como herramientas de enseñanza efectiva. La especificidad de contexto y la necesidad de construir una base de datos adecuada a partir de la cual razonar por vía de la analogía, requiere haber visto muchos ejemplos, que los estudiantes sean capacitados para conectar activamente con el proceso de solución de problemas, y que los ejemplos proporcionen una representación precisa del abanico de presentaciones con las que se presentan las condiciones específicas ${ }^{34}$. Este último criterio se ha hecho cada vez más importante al considerar como la evolución del sistema sanitario en muchas partes del mundo ha disminuido la probabilidad de que los estudiantes encuentren al azar un gran número de algunas condiciones médicas durante sus experiencias de aprendizaje basadas en la clínica. Como resultado, se requiere una mayor conciencia y creatividad por parte de los profesores clínicos y los planificadores del curriculum para asegurar que los estudiantes reciben una exposición adecuada a los casos pedagógicamente útiles.

En segundo lugar, los profesores clínicos deberían reconocer que el abordaje tradicional en dos etapas de la enseñanza clínica, que se remonta como mínimo hasta Flexner ${ }^{39}$, en el que se esperaba que los estudiantes dominaran las ciencias básicas antes de proceder a considerar problemas clínicos, puede ser inadecuado. Hay evidencia que sugiere que una comprensión de los mecanismos de las ciencias básicas puede ayudar a los que diagnostican a generar hipótesis precisas y, por lo tanto, debería permanecer como parte de la formación médica ${ }^{40,41}$. No obstante se debe reconocer, que esta estrategia proporciona una manera de alcanzar el diagnóstico correcto, no la manera. De modo similar, simplemente trabajando en una sala e interaccionando con una serie de pacientes sin una focalización adicional en los principios subyacentes a los casos, puede hacer un flaco favor al debilitar una de las vías por las que puedan ser capaces de llegar a una solución para futuros casos.

En tercer lugar, la práctica con casos debería proceder de una manera que mimetizara el uso even- tual del conocimiento resultante. Los clínicos raramente encuentran un caso nuevo en el que se conozca el diagnóstico. Trabajando con casos de los libros de texto en los que uno conoce el diagnóstico como resultado del capítulo (o el tema de la lectura) no capacita a los estudiantes para determinar si hubieran sido capaces de reconocer el caso si lo hubieran visto en la sala. Si la presentación del paciente y la representación del caso esbozadas en la Figura 3, están plenamente enlazadas con un diagnóstico en particular, la práctica de la fase crítica de comprobación de la hipótesis, se pierde. Apoyando esta afirmación, muchos investigadores han mostrado que es pedagógicamente óptima una "práctica mixta" en la que los estudiantes ven casos de categorías múltiples mezcladas (en oposición a la práctica cerrada en la que los estudiantes trabajan con un bloque de casos de una categoría diagnóstica antes de proceder al siguiente bloque de casos de una categoría diagnóstica diferente $)^{42,43}$.

Además, los profesores clínicos no deberían fiarse de los estudiantes que espontáneamente hacen comparaciones significativas entre problemas. Es mucho más probable que los estudiantes razonen con éxito por vía de la analogía cuando se les ha ordenado explícitamente intentar identificar semejanzas en los conceptos subyacentes de problemas distintos superficialmente ${ }^{44}$. De esta forma, los principios inherentes en nuevos ejemplos se deberían relacionar cuando sea posible, con los principios inherentes en los casos precedentes. Un beneficio adicional de este tipo de estrategia educativa es el producto colateral de proporcionar mejor información para el profesor clínico con relación a cuando los estudiantes puedan estar experimentando dificultades. Es bien conocido que los expertos tienen dificultades para predecir los errores que hacen otros $^{45}$. Proporcionando a los estudiantes una oportunidad para descubrir errores idiosincrásicos capacita a los profesores clínicos para focalizar los esfuerzos de enseñanza en la dirección que sea más probable beneficiar a los estudiantes individuales.

Finalmente, la flexibilidad inherente en el razonamiento clínico y al prevalencia de la especificidad de contexto tiene implicaciones muy reales para los profesores clínicos a la hora de evaluar a los alumnos. Uno no debería asumir que porque un estudiante haya proporcionado un diagnóstico y/o un plan de manejo precisos, esto supone que el estudiante entiende perfectamente los mecanismos fisiológicos subyacentes al proceso. De la misma manera, incluso si el estudiante puede explicar los 
mecanismos fisiológicos subyacentes, uno no debería asumir que se proporcionará un diagnóstico preciso cuando se encuentre con el caso siguiente. En los dominios que son influenciados por la especificidad de contexto (p.ej., todos los dominios), se requiere un enfoque "multi-biopsia" para la evaluación para valorar con precisión la actuación del estudiante. Mientras que nadie soñaría en evaluar conocimientos con una sola pregunta de respuesta múltiple, el reconocimiento de la necesidad de ampliar la muestra cuando se evalúan otras características de la experteza ha sido menos evidente. Retrospectivamente se ha aclarado que la especificidad de contexto es un contribuyente mayor para las pobres cualidades psicométricas de los ejercicios de evaluación como el Triple Salto, Problemas de Manejo de Pacientes y Exámenes orales / escritos largos ${ }^{46}$. Cuanto más tiempo se precisa para realizar una tarea de evaluación menor es la oportunidad que tienen los estudiantes de completar la tarea múltiples veces. Para asegurar la recogida de información que indique fiablemente el nivel de habilidad del estudiante, los profesores clínicos deberían seguir utilizando herramientas como las ECOEs, Ejercicios de Razonamiento Clínico y Pruebas de Elección Múltiple ${ }^{47}$.

\section{AGRADECIMIENTOS}

El autor desea agradecer los comentarios llenos de insight de Lee Brooks, Geoff Norman, y Glenn Regehr proporcionados durante la creación de este manuscrito.

\section{BIBLIOGRAFÍA}

1. Cunnington JPW, Turnbull JM, Regehr G, Marriott M, Norman GR. The effect of presentation order in clinical decision making. Acad Med 1997; 72 (Suppl.): S40-S42.

2. Wilkerson L, Irby DM. Strategies for improving teaching practices: A comprehensive approach to faculty development. Acad Med 1998; 73:387-96.

3. Irby DM. What clinical teachers in medicine need to know. Acad Med 1994; 69:333-42.

4. Bargh JA, Chartrand TL. The unbearable automaticity of being. Amer Psychol 1999; 54: 462-79.

5. Levison ME. Pneumonia, including necrotizing pulmonary infections (lung abscess). In E Braunwald, SL Hauser, AS Fauci, DL Longo, DL Kasper, JL Jameson (Eds). Harrison's Principles of Internal Medicine (15th ed). New York: McGraw-Hill, 2001 (pp. 1475-85).
6. Goldhaber SZ. Pulmonary thromboembolism. In E Braunwald, SL Hauser, AS Fauci, DL Longo, DL Kasper, JL Jameson (Eds). Harrison's Principles of Internal Medicine (15th ed). New York: McGraw-Hill, 2001 (pp.1508-13).

7. Weinstein MD, Fineburg HU, Elstein AS, et al. Clinical decision analysis. Philadelphia, PA: W.B. Saunders, 1980.

8. Wigton RS. Use of linear models to analyze physicians' decisions. Med Dec Making 1988; 8: 241-52.

9. Sackett DL, Haynes RB, Guyatt GH, Tugwell P. Clinical Epidemiology: A Basic Science for Clinical Medicine (2nd ed). Toronto: Little Brown and Company, 1991.

10. Fletcher R, Fletcher S, Wagner E. Clinical Epidemiology: The Essentials (2nd ed). Baltimore: Williams \& Wilkins, 1996.

11. Patel VL, Goren GJ. Knowledge-based solution strategies in medical reasoning. Cog Sci 1986; 10: 91-116.

12. Patel VL, Arocha JF, Kaufman DR. Diagnostic reasoning and medical expertise. Psychol Reason Motiv 1994; 31: 187-252.

13. Genero N, Cantor N. Exemplar prototypes and clinical diagnosis: Toward a cognitive economy. J Soc Clin Psychol 1987; 5: 59-78.

14. Bordage G. Elaborated knowledge: a key to successful diagnostic thinking. Acad Med 1994; 69: 883-5.

15. Mandin H, Jones A, Woloschuk W, Harasym P. Helping students learn to think like experts when solving clinical problems. Acad Med 1997;72: 173-9.

16. Elieson SW, Papa FJ. The effects of various knowledge formats on diagnostic performance. Acad Med 1994; 69 (Suppl.): S81-3.

17. Brooks LR. Decentralized control in categorization: The role of prior processing episodes. In U Neisser (Ed.). Concepts and conceptual development: Ecological and intellectual factors in categorization. Cambridge: Cambridge University Press, 1987 (pp. 141-74).

18. Jacoby LL, Baker JG, Brooks LR. The priority of the specific: Episodic effects in picture identification. J Exp Psychol: Learn Mem Cognit 1989; 15: 275-81.

19. Norman GR, Brooks LR. The non-analytical basis of clinical reasoning. Adv Health Sci Educ 1997; 2: 17384.

20. Brooks LR, Allen SW, Norman GR. Role of specific similarity in a medical diagnostic task. J Exp Psych: Gen 1991; 120: 278-87.

21. Hatala RM, Norman GR, Brooks LR. Influence of a single example on subsequent electrocardiogram interpretation. Teach Learn Med 1999; 11: 110-7.

22. Coderre S, Mandin H, Harasym PH, Fick GH. Diagnostic reasoning strategies and diagnostic success. Med Educ 2003; 37: 695-703. 
23. Neufeld VR, Norman GR, Feightner JW, Barrows HS. Clinical problem-solving by medical students: A crosssectional and longitudinal analysis. Med Ed 1981: 15; 315-22.

24. Eva KW. The aging physician: Changes in cognitive processing and their impact on medical practice. Acad Med 2002; 77 (Suppl.): S1-S6

25. Brooks LR, LeBlanc VR, Norman GR. On the difficulty of noticing obvious features in patient appearance. Psychol Sci. 2000; 11: 112-7.

26. Eva KW, Brooks LR. The underweighting of implicitly generated diagnoses. Acad Med 2000; 75 (Suppl.): S81-S83.

27. Eva KW. The influence of differentially processing information on diagnostic decision-making [dissertation]. McMaster University, Hamilton, Ontario, Canada, 2001.

28. Eva KW, Brooks LR, Norman GR. Forward reasoning as a hallmark of expertise in medicine: Logical, psychological, and phenomenological inconsistencies. In SP Shohov (Ed.). Advances in Psychological Research (Volume 8). New York: Nova Science Publishers, Inc., 2002 (pp. 41-69).

29. Whittlesea BWA, Brooks LR, Westcott C. After the learning is over: Factors controlling the selective application of general and particular knowledge. $\mathrm{J}$ Exp Psychol: Learn Mem Cognit 1994; 20: 259-74.

30. Regehr G, Cline DJ, Norman GR, Brooks LR. Effect of processing strategy on diagnostic skill in dermatology. Acad Med 1994; 69: S34-S36.

31. Kulatunga-Moruzi C, Brooks LR, Norman GR. Coordination of analytic and similarity-based processing strategies and expertise in dermatological diagnosis. Teach Learn Med 2001; 13: 110-6.

32. Ark T, Brooks LR, Eva KW. The best of both worlds: Adoption of a combined (analytic and non-analytic) reasoning strategy improves diagnostic accuracy relative to either strategy in isolation. In preparation.

33. Norman GR, Brooks LR, Colle CL, Hatala RM. The benefit of diagnostic hypotheses in clinical reasoning: Experimental study of an instructional intervention for forward and backward reasoning. Cognit Instruct 2000; 17: 433-48.

34. Eva KW, Neville AJ, Norman GR. Exploring the etiology of content specificity: Factors influencing analogic transfer and problem solving. Acad Med 1998; 73: S1-S5.
35. Eva KW. On the generality of specificity. Med Educ 2003; 37: 587-8.

36. Levy BA, Hinchley J. Individual and developmental differences in the acquisition of reading skills. In $\mathrm{TH}$ Carr, BA Levy (Eds.). Reading and its development: Component skills approaches. San Diego, CA: Academic Press, Inc., 1990 (pp. 81-128).

37. Elstein AS, Shulman LS, Sprafka SA. Medical problem solving: An analysis of clinical reasoning. Cambridge, MA: Harvard University Press, 1978.

38. Custers EJFM, Regehr G, Norman GR. Mental representations of medical diagnostic knowledge: A review. Acad Med 1996; 71 (Suppl.): S24-S26.

39. Flexner A. Medical education in the United States and Canada: A report to the Carnegie Foundation for the Advancement of Teaching. Boston, MA: The Carnegie Foundation for the Advancement of Teaching, 1910.

40. Schmidt HG, Norman GR, Boshuizen HP. A cognitive perspective on medical expertise: theory and implication. Acad Med 1990; 65: 611-21.

41. Woods NN, Brooks LR, Norman GR. The Value of Basic Science in Clinical Diagnosis: Creating Coherence among Signs and Symptoms. Submitted manuscript.

42. McKenzie CRM. Taking into account the strength of an alternative hypothesis. J Exper Psych: Learn, Mem, Cognit 1998; 24: 771-92.

43. Hatala RM, Brooks LR, Norman GR. Practice makes perfect: the critical role of mixed practice in the acquisition of ECG interpretation skills. Adv Health Sci Educ 2003; 8: 17-26.

44. Thompson L, Gentner D, Loewenstein J. Avoiding missed opportunities in managerial life: Analogical learning improves case-based transfer. Organ Behav Human Dec Process 2000; 47: 98-123.

45. Norman GR, Brooks LR, Rosenthal D, Allen SW, Muzzin LJ. The development of expertise in dermatology. Arch Derm 1989; 125: 1063-8.

46. Nendaz MR, Tekian A. Assessment in problem-based learning medical schools: A literature review. Teach Learn Med 1999; 11: 232-43.

47. Swanson D, Norman GR, Linn RI. Performance based assessment: Lessons from health professions. Educ Res 1995; June: 5-12. 